\title{
La evaluación de un programa de formación con profesorado de escuelas de personas adultas del Perú
}

\author{
María Jesús Perales ${ }^{\mathrm{a}}$, Carlos Sancho-Álvarez ${ }^{2}$ y Margarita Bakieva ${ }^{3}$ \\ Universitat de València ${ }^{123}$ \\ Orcid ID: http://orcid.org/0000-0003-2033-27501 \\ Orcid ID: https://orcid.org/0000-0001-9489-2502² \\ Orcid ID: http://orcid.org/0000-0002-2716-0755³
}

\section{Recibido: 13 de noviembre de $2017 \quad$ Aceptado: 20 de diciembre de 2017}

\section{Resumen}

Se presenta la evaluación de un programa de formación con profesorado de Escuelas de Personas Adultas. El Seminario en Gestión Pedagógica para Directores, Subdirectores y Docentes nombrados de los Centros de Educación Básica Alternativa Públicos se desarrolla en España desde la Universitat de València. El programa se diseña a partir de un proceso consensuado entre las instituciones y autoridades implicadas, vinculando un adecuado análisis de necesidades previo. Asimismo, el seminario se diseña combinando tres aproximaciones de formación: conferencias sobre temáticas específicas, visitas a lugares y diferentes recursos educativos y cursos formativos. Diseñar un programa de formación sobre educación de adultos para un colectivo específico, a partir de su implantación, debe ser analizado y evaluado para poder destacar elementos y aspectos a mejorar. Así como analizar las características que han funcionado adecuadamente o han aparecido como imprevisto en el desarrollo. Para ello, se trabaja con los y las 99 docentes de EBA en activo, participantes en el seminario, para que puedan realizar un análisis desde diferentes enfoques para poder aproximar resultados y datos. Se desarrolla una metodología mixta con análisis estadísticos sobre datos cuantitativos y análisis de contenido sobre resultados cualitativos, a partir de un estudio de encuesta, murales y exposición de fotografías. Los resultados, en general, son adecuados en ambos sentidos en todos los casos estudiados. La evaluación de producto realizada ha sido muy enriquecedora para poder tomar decisiones posteriores a su realización y seguir avanzando en la mejora de este tipo de programas de formación de profesorado.

Palabras clave: evaluación de programas, educación de personas adultas, profesorado, Perú, España. 


\title{
The evaluation of a training program for teachers in adult education in Peru
}

\begin{abstract}
We present the evaluation of a training program with teachers of adult education. The Seminario en Gestión Pedagógica para Directores, Subdirectores y Docentes nombrados de los Centros de Educación Básica Alternativa Públicos is conducted in Spain from the University of Valencia. The program is designed based on a consensus process between the institutions and authorities involved, linking an adequate analysis of previous needs. Likewise, the seminar is designed combining three training approaches: lectures on specific topics, visits to places and different educational resources and training courses. Designing a training program on adult education for a specific group, from its implementation, should be analyzed and evaluated to highlight elements and aspects to improve, as well as analyzing the characteristics that have worked properly or have appeared as unexpectedly in the development process. To do this, this study worked with the 99 teachers of EBA active, participants in the seminar, so they could perform an analysis from different approaches to approximate results and data. A mixed methodology was developed with statistical analysis on quantitative data and content analysis on qualitative results, based on a survey study, murals and photography exhibition. The results, in general, are adequate in both ways in all the cases studied. The evaluation of the product has been very enriching to be able to make decisions after its realization and to continue advancing in the improvement of this type of teacher training programs.
\end{abstract}

Key-words: program evaluation, adult education, teachers, Peru, Spain.

\section{Introducción ${ }^{b}$}

La EBA (Educación Básica Alternativa) en Perú, así como en el mismo contexto de la EPJA (Educación de Personas Jóvenes y Adultas), la EC (Educación Comunitaria) o la ETP (Enseñanza Técnica y Profesional), se relacionan con el margen y hacia los y las sujetos históricamente excluidos de la modalidad EBR (Educación Básica Regular). Por ello, de manera específica la Educación Básica Alternativa se relaciona en muchos casos con procesos de retorno o fracaso escolar, ya que cuenta con la atención hacia diferentes colectivos en riesgo de dificultad escolar. Es decir, jóvenes, adolescentes y adultos/as que anteriormente no pudieron acabar los estudios obligatorios, o que simplemente no se matricularon o no pudieron acceder por diferentes motivos (Venegas, 2015).

Ante este panorama, el profesorado ha de actuar a partir de su formación y experiencia, desarrollando metodologías que atiendan a toda la diversidad y que puedan generar procesos de enseñanza-aprendizaje para todos y para todas. Además, el profesorado debe enriquecerse de los recursos formativos y/o institucionales que están a

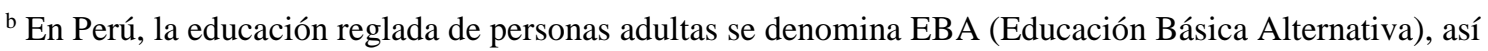
como las escuelas donde se imparte esta modalidad se conocen como CEBA (Centro de Educación Básica Alternativa) (Venegas, 2015).
} 
su alcance; a través de programas de formación, como el que presentamos objeto de estudio.

El educador debe ser una ayuda fundamental para que el discente pueda hacer posible su proyecto personal de vida, sin olvidar la dimensión ética sobre la evaluación que ejerce en relación a su labor docente, ya que, si nos preocupamos por adecuar su complejidad metodológica, con ello podremos evitar errores de juicio y nos comprometeremos adecuadamente (Jornet, 2007). Por lo tanto, un aspecto fundamental sobre la formación del profesorado, así como en relación a la posible evaluación de programas socio-educativos.

Si bien por función corresponde al MINEDU la adopción de medidas con visión estratégica, este esfuerzo debe involucrar necesariamente a directivos y docentes que laboran en EBA, sin cuyo aporte, compromiso y calificación difícilmente podrán plasmarse los cambios requeridos. Desde la sociedad civil corresponde alentar y acompañar este proceso (Paiba, 2015, p. 42).

Para atender esta demanda, desde la administración educativa del Perú se diseña con la Universitat de València un programa intensivo de formación para docentes EBA, de un mes de duración, a desarrollar en la ciudad de Valencia, con docentes de la Universitat.

El programa se diseña combinando tres aproximaciones de formación: las conferencias sobre temáticas específicas, las visitas a lugares y recursos educativos considerados relevantes y los cursos propiamente dichos. Esta triple aproximación permite combinar, de una forma equilibrada, el acercamiento sistemático a los contenidos seleccionados como relevantes, con una aproximación al contexto cultural, organizativo y educativo de Valencia y la Comunidad Valenciana, permitiendo conocer de primera mano propuestas y experiencias vinculadas con la educación de adultos.

Los cursos específicos se organizan en torno a once temáticas concretas:

- $\quad$ Bases para la educación de personas jóvenes y adultas.

- Políticas educativas y documentos internacionales.

- $\quad$ Evaluación en educación de personas jóvenes y adultas.

- Diagnóstico y evaluación de proyectos.

- Teorías del aprendizaje.

- Alfabetización. 
- Enfoque de competencias y currículum.

- Estrategias de enseñanza-aprendizaje: aprendizaje basado en problemas, aprendizaje expositivo, aprendizaje cooperativo.

- Materiales didácticos. Creación y análisis.

- $\quad$ Estilos de aprendizaje. Inteligencia emocional.

- $\quad$ Estilos de aprendizaje. Inteligencias múltiples.

- $\quad$ Diseño de proyecto. Taller de proyecto.

El curso de Diseño de proyecto toma un protagonismo especial en el programa, al ser un elemento articulador y contextualizador del aprendizaje, que contribuye a hacerlo significativo, y a facilitar el establecimiento de redes locales de colaboración sostenibles en el tiempo, siendo un curso que se desarrolla de forma longitudinal a lo largo de todo el programa intensivo.

Un programa intensivo como el planteado tiene la ventaja de concentrar la experiencia de aprendizaje, y de vincularla al conocimiento de otras experiencias y contextos, en este caso los de Valencia y la Comunidad Valenciana. Pero puede tener el riesgo de la artificialidad y la descontextualización. Por ello, desde el diseño del programa de formación se plantea el taller de proyecto, para configurar equipos de trabajo integrados por docentes de EBA de regiones cercanas, que puedan realizar un análisis de las necesidades de sus contextos y desde allí plantear el diseño de un proyecto en el que, en torno a un tema, se integren las competencias, los contenidos y los aprendizajes que se van planteando a lo largo del programa intensivo de formación. El papel de los y las docentes de la Universiat de València, como facilitadores y orientadores del desarrollo del trabajo en los grupos, se configura como elemento de enlace entre las diferentes realidades y como vínculo sistemático con los contenidos del programa intensivo de formación, sin interferir en el protagonismo de cada uno de los grupos en la creación de su dinámica de colaboración y de su proyecto concreto.

\section{La evaluación como garantía de calidad de los programas}

La propuesta de un programa de formación tiene en la evaluación una herramienta clave para acompañar todo su proceso, permitiendo garantizar la referencia a la calidad en cada una de las fases. 
En este sentido, propuestas metodológicas como la de Stufflebeam y Skinfield (1987) son especialmente útiles porque vinculan el ciclo del programa a la estrategia de evaluación, de forma que ésta se convierte en referente para el diseño, para el desarrollo y para la valoración final del mismo. Por otro lado, el carácter sistemático pero abierto de la propuesta permite integrar en la misma aportación de diferentes modelos de evaluación, así como técnicas de recogida de información vinculadas a aproximaciones cuantitativas y/o cualitativas, en función de la finalidad y el objeto de la evaluación (Jornet, GonzalezSuch y Perales, 2013).

Diseñar un programa de formación sobre educación de adultos para un colectivo específico, como el profesorado de EBA de Perú, debe ser un proceso consensuado entre las autoridades implicadas, y vinculado con un análisis de necesidades bien especificado. Este análisis de necesidades es lo que Stufflebeam llama evaluación del contexto.

El programa propuesto (en términos de competencias a desarrollar, de contenidos a abordar, de metodologías didácticas y de trabajo seleccionadas, de sistemas de seguimiento y evaluación de los y las participantes, de garantías de continuidad y sostenibilidad en el contexto...) debe responder a las necesidades identificadas en el estudio previo; necesidades que se pueden vincular tanto con los objetivos perseguidos por la administración educativa peruana (que encarga el proceso formativo), como con las necesidades percibidas por el profesorado EBA, y con las prioridades planteadas desde la entidad formadora, en este caso la Universitat de València, en España.

Las estrategias que se utilicen para ello pueden ser muy variadas, optando por implicar a las audiencias de formas muy diversas. Estrategias directivas, planteadas desde la lógica de un encargo institucional, son tan viables como estrategias mucho más participativas, donde se conciba el propio proceso de evaluación del contexto como parte indirecta del proceso formativo, puesto que contribuye al diseño del propio proyecto. Nuevamente, la apertura y sistematicidad del modelo de Stufflebeam permite incorporar estas diferentes perspectivas.

Una vez planteada la propuesta de programa, el modelo de Stufflebeam prevé en la evaluación de entrada un análisis de la misma para valorar su adecuación como respuesta a la evaluación de contexto realizada. Esta evaluación de entrada permitirá 
Identificar y valorar la capacidad del sistema, las estrategias del programa, las alternativas, la planificación de procedimientos para llevar a cabo las estrategias, los presupuestos y los programas (Jiménez, 1999, p. 49).

Por un lado, esta evaluación de entrada permite analizar la propuesta de programa desde criterios como su pertinencia, su suficiencia o su calidad técnica (Pérez-Juste, 2000; Perales, Ortega y Jornet, 2012), valorando si realmente es una propuesta que ajusta a los requerimientos, y, desde una perspectiva formativa, introduciendo en la misma las mejoras que se identifiquen como relevantes a la luz del análisis realizado.

Así mismo, esta evaluación de entrada se vincula con los procesos iniciales del programa, el establecimiento de posibles líneas base, la selección de participantes, la valoración de participantes y formadores, etc. Se trata de valorar los elementos de inicio del programa.

Vinculado con este planteamiento, en la gestión del programa de formación para docentes EBA desarrollado en la Universitat de Valencia se incorpora, a petición de las autoridades peruanas, el diseño y desarrollo de una prueba de selección de candidatos, que permita seleccionar al grupo final de becarios entre los y las postulantes. La prueba se diseña desde los requerimientos del modelo criterial (Jornet y González-Such, 2009), estableciendo un nivel mínimo exigido para participar en el curso, que se combina con referentes normativos, al deber ofrecer un listado final de 100 participantes. La aplicación on-line $^{c}$ de la misma, vinculada con un sistema de claves personalizas, ofreció las necesarias características de factibilidad y seguridad al proceso de evaluación de sujetos.

El inicio de la implantación del programa supone pasar a la tercera evaluación propuesta por Stufflebeam, la evaluación de proceso. Esta evaluación acompaña el desarrollo del programa, apoyando la toma de decisiones vinculada con las mejoras necesarias para la optimización del mismo, combinando exigencias de respeto a la planificación y de flexibilidad en el proceso. Esta evaluación, con clara finalidad formativa, es especialmente importante en programas que se alargan en el tiempo, puesto

`Se realizó a través de una encuesta en línea utilizando el programa LimeSurvey, adaptado al área educativa por el grupo de innovación educativa InnovaMide http://www.uv.es/innovamide/ para facilitar la administración y recopilación de datos en relación con cada criterio de evaluación(Dray, Lowenthal, Miszkiewicz, Ruiz-Primo y Marczynski, 2011). 
que la experiencia de valoración es más intensa, y el desarrollo temporal permite una mejor identificación e incorporación de propuestas de mejora.

Al finalizar el programa es el momento de abordar la cuarta evaluación del modelo, la evaluación de producto. Esta evaluación permite tomar decisiones sobre "la continuación, finalización, modificación o readaptación de la actividad del cambio. Y presentar un informe claro de los efectos (deseados y no deseado, positivos y negativos)" (Jiménez, 1999, p. 49). Entre los criterios que se pueden utilizar destacan los de eficacia, eficiencia, satisfacción de los implicados, impacto y funcionalidad (Perales, Ortega y Jornet, 2013).

Este trabajo se centra fundamentalmente en la metodología utilizada en la evaluación de producto, o evaluación final, del programa de formación para docentes EBA del Perú desarrollado en la Universitat de València, así como en los resultados de dicha evaluación. Ambas serán presentadas más adelante.

\section{Grupo de participantes}

Se trata de un grupo de 99 docentes en activo, con una media de edad de 49 años, distribuyéndose el 53\% hombres y el $47 \%$ mujeres. A continuación, se describe el grupo de estudio de acuerdo a diferentes variables explicativas y características sociodemográficas.

El profesorado implicado en el curso de formación recibido, sin duda es diverso. Su procedencia es en torno a todo el país de Perú y trabajan en diferentes escuelas y zonas.

Asimismo, se puede observar su distribución de acuerdo a la región donde trabaja actualmente y el tipo de zona donde está su CEBA -ver gráfico 1-.

Gráfico 1. Distribución de la muestra sobre región y zona 


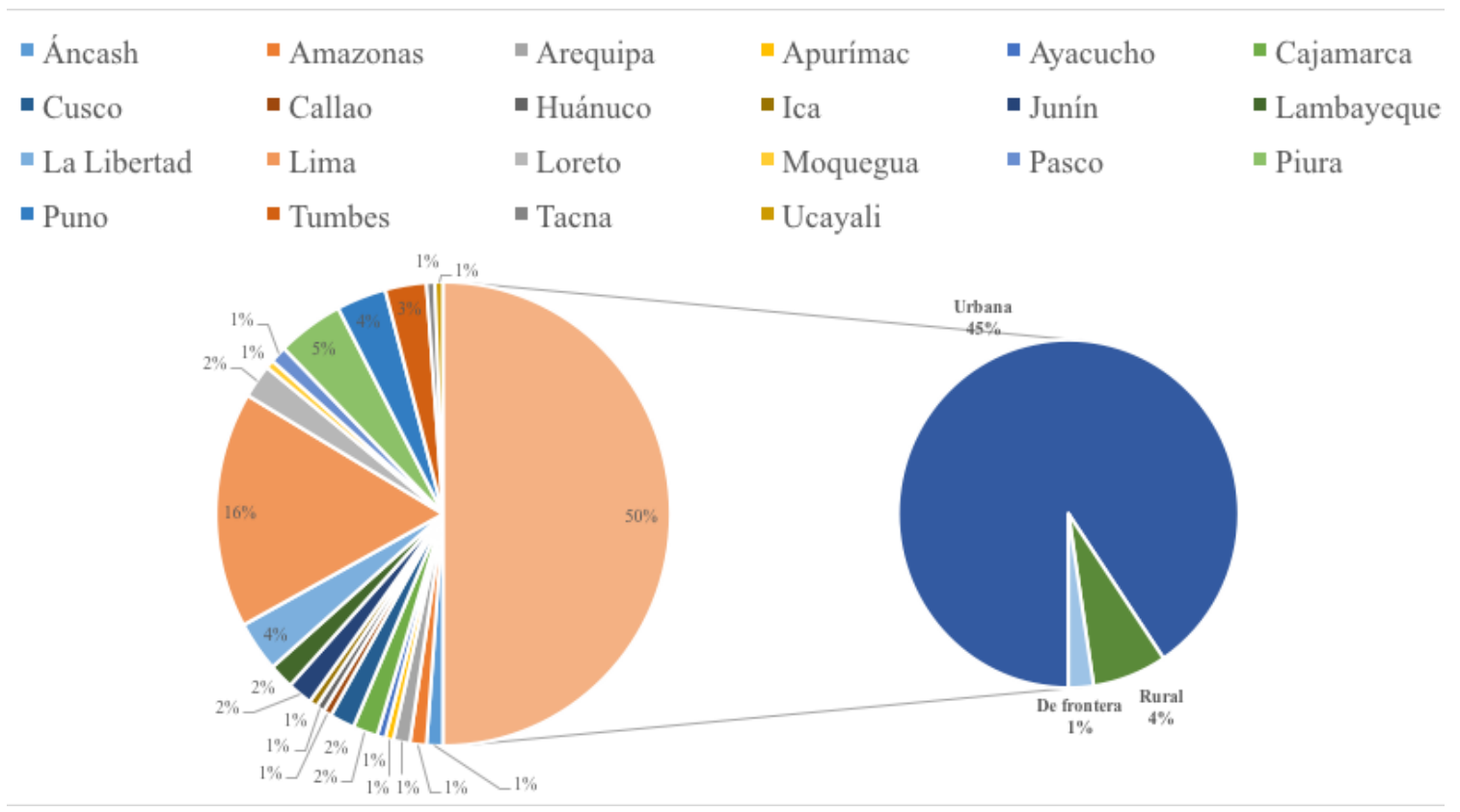

Asimismo, el profesorado participante cuenta con formación como profesor/a de educación primaria (13\%) y como profesor/a de educación secundaria (87\%), distribuyéndose su labor docente sobre dos especialidades educativas generales: Ciencias $(53 \%)$ y Humanidades (43\%). En cuanto al ciclo donde se desarrolla su trabajo se relaciona con el Intermedio en un $12 \%$ y Avanzado en $88 \%$; realizando una atención presencial (82\%) y semipresencial (18\%).

La experiencia socio-profesional del profesorado en tiempo de servicio en la modalidad EBA es amplia, distribuida en un promedio de 14 años, siendo el mínimo de 5 años y el máximo de 29 años. Así como un tiempo de servicio total como docentes de media 24 años (mínimo 12 años y máximo 35 años).

A continuación, se puede observar su distribución de acuerdo al área de desarrollo profesional y dónde desarrolla su función docente -ver gráfico 2-.

Gráfico 2. Distribución de la muestra sobre área y función 


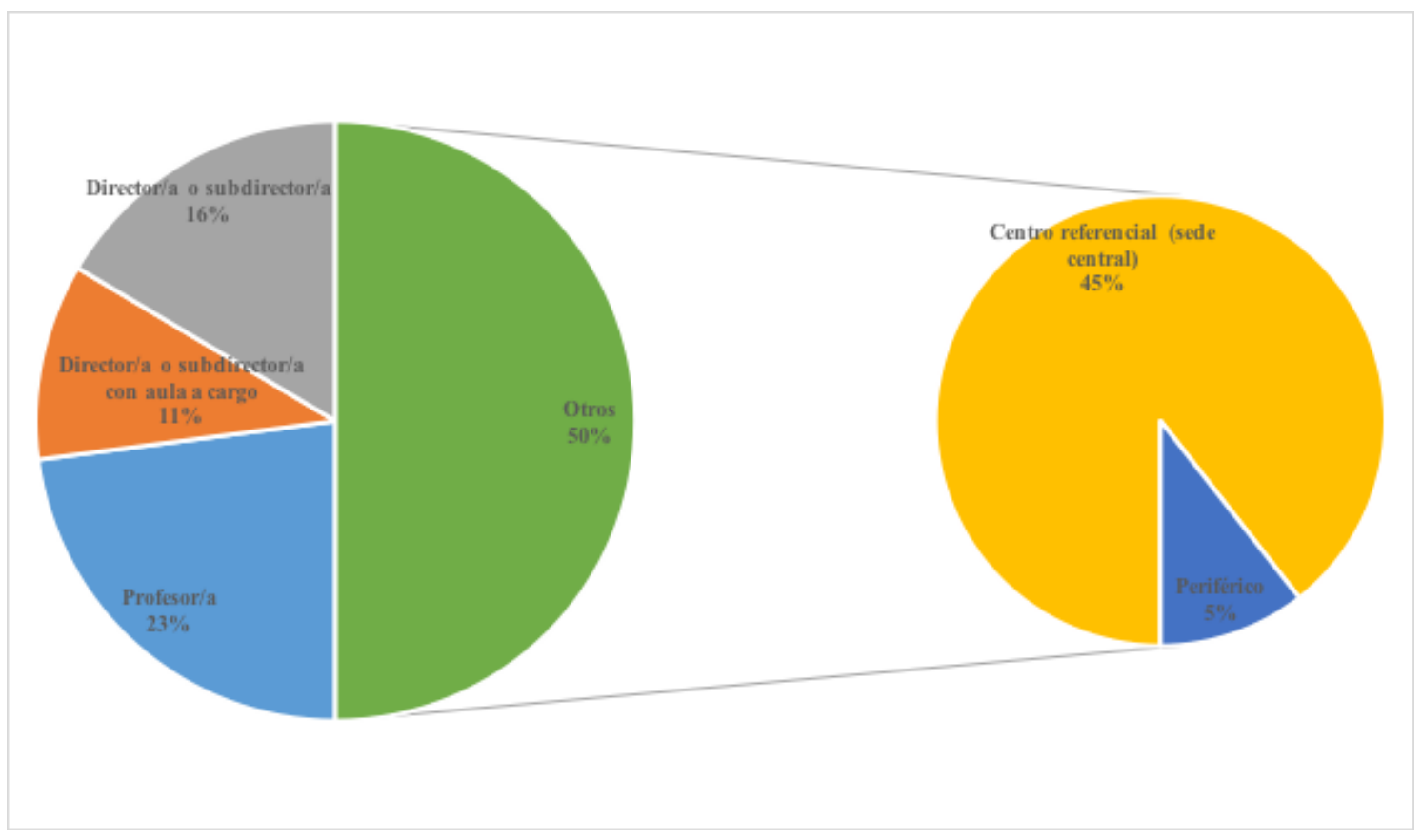

El trabajo de formación que realizan los docentes implicados lo desarrollan en CEBAs con estudiantes de edades comprendidas entre 9 años y 83 (un promedio de edad de 22 años).

Por lo que, la diversidad es amplia en relación al profesorado que realiza su labor docente, así como también los grupos de estudiantes con los que trabajan son muy heterogéneos.

\section{Evaluación de producto}

Con la finalidad de evaluar la efectividad de dicho programa de manera sumativa, así como su adecuación al final de la implantación y desarrollo, que permita una lectura formativa (Scriven, 1967), se apuesta por la complementariedad metodológica en el diseño de la evaluación (Jornet, González-Such y Perales, 2013).

Consideramos que es fundamental la complementariedad metodológica para poder garantizar un mayor y un mejor acercamiento a la realidad estudiada, ya que parece viable combinar la metodología cualitativa y la cuantitativa dentro de una misma investigación, de manera que se pueda sostener la complementariedad entre métodos (Bericat, 1998).

En este sentido, a partir de una encuesta administrada de manera online a todo el profesorado participante, se obtiene información relevante. Asimismo, se desarrolla una sesión presencial donde los docentes implicados tienen la oportunidad de construir cinco 
murales de valoración grupal sobre diferentes aspectos del programa, así como de presentar una fotografía de manera individual, que haya sido relevante para cada uno/a de ellos/as. En este caso, se ha pretendido llevar a cabo una evaluación creativa de acuerdo a algunas estrategias contemporáneas para representar el aprendizaje (SánchezRodríguez, 2015).

A partir de la información recabada mediante el cuestionario online se han realizado los siguientes análisis de datos:

○ Análisis estadísticos descriptivos sobre medidas de tendencia central y dispersión.

○ Prueba W de Kendall

- Análisis de diferencias entre variables

Por otro lado, se ha empleado la técnica de análisis de contenido mediante un sistema categorial inductivo de la información, para cada uno de las conclusiones recogidas sobre los murales y las fotografías resultantes.

\section{Instrumentos}

Para facilitar la recogida de información, la opinión y valoración del profesorado implicado en el periodo formativo, se ha diseñado y construido un cuestionario de satisfacción de acuerdo a tres dimensiones: (1) Actividades y experiencias, (2) Satisfacción general y (3) Aportaciones.

A continuación, se presenta el conjunto de ítems de acuerdo a cada dimensión:

\section{Dimensión 1. Actividades y experiencias:}

- Su relevancia respecto a mi desarrollo como docente EBA.

- Su aplicabilidad en el contexto del CEBA.

- El carácter innovador de contenidos y estrategias.

- Su relevancia respecto a mi desarrollo como docente EBA.

- Su aplicabilidad en el contexto del CEBA.

- El carácter innovador de contenidos y estrategias.

- Bases para la educación de personas jóvenes y adultas

- Políticas educativas y documentos internacionales

○ Evaluación en educación personas jóvenes y adultas

○ Diagnóstico y evaluación de proyectos

- Teorías de aprendizaje

○ Alfabetización 
- Enfoque de competencias y currículum

○ Estrategias e-a. APB / Expositivo / Cooperativo

- Materiales didácticos. Creación y análisis

- Estilos de aprendizaje. Inteligencia emocional

- Estilos de aprendizaje. Inteligencias múltiples

○ Diseño de proyecto. Taller de proyecto

\section{Dimensión 2. Satisfacción general:}

○ 2.1 El horario de trabajo para cada día (de 9.00 a 19.30 horas)

○ 2.2 El horario total semanal.

- $2.3 \mathrm{La}$ adecuación de las aulas y espacios en la Facultad

- 2.4 La posibilidad de tener seminarios tutorizados los sábados en aulas de la Facultad.

○ 2.5 El trabajo y coordinación a través de Aula Virtual.

○ 2.6 La resolución de incidencias informáticas.

○ 2.7 La disponibilidad de un bono de transporte para la ciudad.

○ $2.8 \mathrm{El}$ alojamiento en el Colegio Mayor y los apartamentos.

- $2.9 \mathrm{La}$ atención recibida a través del Seguro médico.

○ 2.10 La ciudad de Valencia como entorno de formación.

○ 2.11 La metodología docente en las sesiones ha sido adecuada

○ 2.12 La actitud y la interacción con los docentes han facilitado los aprendizajes y la participación.

○ $2.13 \mathrm{El}$ diseño de proyecto como proceso de aprendizaje cooperativo

○ $2.14 \mathrm{El}$ proceso de acompañamiento del proyecto por docentes UV

○ 2.15 La defensa del proyecto como elemento de socialización

- 2.16 El desarrollo de la Beca como experiencia de aprendizaje me ha aportado elementos que claramente pueden mejorar mi desarrollo como docente de EBA.

○ 2.17 Los elementos aportados desde el Seminario pueden ser transferidos al contexto de mi CEBA.

○ 2.18 Las redes de trabajo creadas en este viaje de aprendizaje han potenciado los aprendizajes desarrollados durante el Seminario.

○ 2.19 Las redes de trabajo generadas en este viaje de aprendizaje podrán potenciar el desarrollo de los CEBAs en el Perú.

\section{Dimensión 3. Aportaciones:}

○ ¿Qué elementos destacarías como muy positivos?, ¿qué aprendizajes más destacados?

○ ¿Qué sugerencias de mejora podrías aportar?

Las dimensiones 1 y 2 del cuestionario se diseñan como escalas tipo Likert, con una escala de 4 puntos, donde 1 es la puntuación mínima y 4 es la puntuación máxima, permitiendo un análisis cuantitativo de las mismas. 
La dimensión 3 introduce la aproximación cualitativa, al ofrecer preguntas abiertas en torno al referente de aportaciones, y desde dos lógicas complementarias: la identificación de elementos positivos y el planteamiento de sugerencias de mejora. El análisis de las preguntas de esta dimensión se basa en la categorización de respuestas para identificar los elementos con mayor frecuencia.

Esta aproximación cualitativa se complementa con una dinámica propiamente participativa, que se desarrolla en cada uno de los dos grupos que se configuraron con los becarios EBA. Tras una primera fase de identificación de elementos clave y de síntesis de ideas (a través de la formulación de las mismas en un número limitado de post-its), se organizó una puesta en común participativa en torno a cuatro ejes clave, visualizados en murales que se construyeron colaborativamente. Paralelamente, se propuso una dinámica de selección por cada uno de los participantes de una imagen representativa del programa formativo, y elegida entre la multitud de fotografías que cada cual había realizado a lo largo de mes de formación. La combinación de estas estrategias, y la dinámica de asamblea generada en torno a las misas, ofreció una síntesis significativa de los elementos considerados destacados desde la subjetividad de los y las participantes.

\section{Resultados}

Los resultados obtenidos en relación a la evaluación del programa de formación a partir de la opinión de los y las participantes, se presenta, con respecto a diferentes bloques temáticos en torno a cuatro puntos de interés, desarrollados en cuanto al cuestionario de satisfacción y la valoración creativa personal y grupal.

$\checkmark$ Resultados descriptivos valoraciones cuantitativas (una escala del 1 al 4 puntos, donde 1 es la puntuación mínima y el 4 es la máxima)

$\checkmark$ Resultados análisis estadísticos valoraciones cuantitativas

$\checkmark$ Valoraciones cualitativas (preguntas abiertas)

$\checkmark$ Valoraciones creativas personales (murales y fotografías)

\subsection{Resultados descriptivos. Valoraciones cuantitativas (1)}

En la valoración del programa se han considerado las tres estrategias de trabajo del programa integradas en el boque temático 1:

- las conferencias sobre temáticas específicas

- las visitas a lugares y recursos educativos considerados relevantes 
- los cursos en las aulas de la Universitat de València

Dentro del bloque 1 de preguntas se presentan los resultados de acuerdo a la valoración de cada tipo de actividades. Se recuerda que la escala es de cuatro puntos, siendo 1 la valoración más baja (muy en desacuerdo) y 4 la más alta (muy de acuerdo).

Tabla 1. Resultados descriptivos bloque 1. Actividades conferencias y experiencias

\begin{tabular}{llllll} 
Ítems respecto a las conferencias: & $\mathrm{N}$ & $\mu$ & $\sigma$ & $\mathrm{CV}$ \\
\hline 1.1 Su relevancia respecto a mi desarrollo como docente EBA. & 88 & 3,45 & 0,66 & 19,09 \\
1.2 Su aplicabilidad en el contexto del CEBA. & 88 & 3,28 & 0,64 & 19,56 \\
1.3 El carácter innovador de contenidos y estrategias. & 87 & 3,46 & 0,66 & 19,11 \\
\hline & 88 & 3,39 & 0,54 & 19,11 \\
\hline & & & & \\
Ítems respecto a las experiencias educativas mostradas en las visitas: & 88 & $\mathbf{3 , 6 1}$ & 0,58 & 15,93 \\
1.4 Su relevancia respecto a mi desarrollo como docente EBA. & 88 & 3,32 & 0,74 & 22,17 \\
1.5 Su aplicabilidad en el contexto del CEBA. & 88 & $\mathbf{3 , 5 8}$ & 0,62 & 17,32 \\
1.6 El carácter innovador de contenidos y estrategias. & 88 & 3,50 & 0,55 & 19,113 \\
t & &
\end{tabular}

La valoraciones más altas en cuanto a las actividades de conferencias y experiencias son en relación al ítem 1.4 (Su relevancia respecto a mi desarrollo como docente EBA) e ítem 1.6 (El carácter innovador de contenidos y estrategias). Aunque en general, todas son adecuadas ya que superan en todos los casos los 3 puntos.

Tabla 2. Resultados descriptivos bloque 1. Cursos

\begin{tabular}{|c|c|c|c|c|c|c|c|c|}
\hline \multirow[t]{2}{*}{ Ítems respecto a los cursos: } & \multicolumn{4}{|c|}{ Relevancia } & \multicolumn{4}{|c|}{ Transferibilidad } \\
\hline & $\mathrm{N}$ & $\mu$ & $\sigma$ & $\mathrm{CV}$ & $\mathrm{N}$ & $\mu$ & $\sigma$ & $\mathrm{CV}$ \\
\hline $\begin{array}{l}\text { 1.7 Bases para la educación de personas jóvenes } \\
\text { y adultas }\end{array}$ & 85 & 3,46 & 0,59 & 17,02 & 87 & 3,15 & 0,67 & 1,40 \\
\hline $\begin{array}{l}1.8 \text { Políticas educativas y documentos } \\
\text { internacionales }\end{array}$ & 86 & 3,30 & 0,67 & 20,28 & 88 & 3,01 & 0,73 & 24,40 \\
\hline $\begin{array}{l}\text { 1.9 Evaluación en educación personas jóvenes y } \\
\text { adultas }\end{array}$ & 86 & 3,56 & 0,54 & 15,31 & 87 & 3,34 & 0,61 & 18,14 \\
\hline 1.10 Diagnóstico y evaluación de proyectos & 85 & 3,62 & 0,58 & 15,92 & 87 & 3,67 & 0,56 & 15,38 \\
\hline 1.11 Teorías de aprendizaje & 85 & 3,66 & 0,63 & 17,16 & 87 & 3,57 & 0,64 & 17,91 \\
\hline 1.12 Alfabetización & 86 & 3,44 & 0,78 & 22,55 & 88 & 3,26 & 0,86 & 26,50 \\
\hline 1.13 Enfoque de competencias y currículum & 86 & 3,49 & 0,68 & 19,54 & 88 & 3,40 & 0,69 & 20,22 \\
\hline $\begin{array}{l}1.14 \text { Estrategias e-a. APB / Expositivo / } \\
\text { Cooperativo }\end{array}$ & 86 & 3,67 & 0,62 & 16,93 & 88 & 3,61 & 0,67 & 18,50 \\
\hline 1.15 Materiales didácticos. Creación y análisis & 86 & 3,40 & 0,67 & 19,84 & 87 & 3,30 & 0,75 & 22,70 \\
\hline $\begin{array}{l}\text { 1.16 Estilos de aprendizaje. Inteligencia } \\
\text { emocional }\end{array}$ & 85 & 3,79 & 0,56 & 14,74 & 88 & 3,74 & 0,60 & 15,96 \\
\hline $\begin{array}{l}1.17 \text { Estilos de aprendizaje. Inteligencias } \\
\text { múltiples }\end{array}$ & 86 & 3,72 & 0,55 & 14,66 & 88 & 3,67 & 0,64 & 17,39 \\
\hline 1.18 Diseño de proyecto. Taller de proyecto & 85 & 3,74 & 0,52 & 13,77 & 87 & 3,68 & 0,54 & 14,66 \\
\hline $\mathrm{t}$ & 86 & 3,57 & 0,40 & 13,77 & 88 & 3,44 & 0,41 & 13,77 \\
\hline
\end{tabular}


En relación a los resultados descriptivos para el bloque 1 sobre cursos, observamos que los datos más altos se sitúan, en cuanto a relevancia y también en el caso de transferibilidad, en los ítems 1.16 (Estilos de aprendizaje. Inteligencia emocional) y 1.18 (Diseño de proyecto. Taller de proyecto). Sin embargo, cabe destacar que en el resto de situaciones también han resultado datos adecuados ya que todos superan los 3 puntos.

En cuanto al bloque temático 2 se han considerado para su valoración un grupo de ítems sobre elementos contextuales de recursos, y otro, sobre elementos contextuales referidos a cuestionares metodológicos didáctico-pedagógicos.

Tabla 3. Resultados descriptivos bloque 2. Satisfacción general. Elementos contextuales. Recursos

\begin{tabular}{|lllll|}
\hline Ítems respecto a elementos contextuales y recursos: & $\mathrm{N}$ & $\mu$ & $\sigma$ & $\mathrm{CV}$ \\
\hline 2.1 El horario de trabajo para cada día (de 9.00 a 19.30 horas) & 88 & 2,70 & 0,95 & 35,08 \\
2.2 El horario total semanal. & 88 & 2,64 & 0,98 & 37,35 \\
2.3 La adecuación de las aulas y espacios en la Facultad & 65 & 3,51 & 0,69 & 19,60 \\
2.4 La posibilidad de tener seminarios tutorizados los sábados en aulas de la & 84 & 3,08 & 0,87 & 28,12 \\
Facultad. & & & \\
2.5 El trabajo y coordinación a través de Aula Virtual. & 88 & 3,41 & 0,69 & 20,20 \\
2.6 La resolución de incidencias informáticas. & 88 & 3,19 & 0,68 & 21,16 \\
2.7 La disponibilidad de un bono de transporte para la ciudad. & 88 & $\mathbf{3 , 8 8}$ & 0,37 & 9,43 \\
\hline 2.8 El alojamiento en el Colegio Mayor y los apartamentos. & 88 & 3,56 & 0,68 & 19,00 \\
2.9 La atención recibida a través del Seguro médico. & 74 & 2,36 & 1,05 & 44,58 \\
2.10 La ciudad de Valencia como entorno de formación. & 87 & $\mathbf{3 , 8 0}$ & 0,48 & 12,57 \\
t & 88 & 3,22 & 0,41 & 13,77
\end{tabular}

En cuanto a la satisfacción general sobre elementos contextuales y recursos, las valoraciones en general han sido adecuadas. En todos los casos se superan los tres puntos, exceptuando el ítem 2.1 (med 2,7), 2.2 (med 2,64) y el 2.9 (media 2,36) sobre horarios y atención médica. En este sentido, los ítems en los que se encuentran las valoraciones más altos son el 2.7 (La disponibilidad de un bono de transporte para la ciudad) y el 2.10 (La ciudad de Valencia como entorno de formación).

Tabla 4. Resultados descriptivos bloque 2. Satisfacción general. Elementos contextuales. Metodología

\begin{tabular}{|c|c|c|c|c|}
\hline Ítems respecto a elementos contextuales metodología: & $\mathrm{N}$ & $\mu$ & $\sigma$ & $\mathrm{CV}$ \\
\hline 2.11 La metodología docente en las sesiones ha sido adecuada & 88 & 3,45 & 0,62 & 18,05 \\
\hline $\begin{array}{l}2.12 \mathrm{La} \text { actitud y la interacción con los y las docentes han facilitado los } \\
\text { aprendizajes y la participación. }\end{array}$ & 88 & 3,66 & 0,58 & 15,99 \\
\hline 2.13 El diseño de proyecto como proceso de aprendizaje cooperativo & 88 & 3,72 & 0,50 & 13,50 \\
\hline 2.14 El proceso de acompañamiento del proyecto por docentes UV & 88 & 3,64 & 0,57 & 15,70 \\
\hline 2.15 La defensa del proyecto como elemento de socialización & 87 & 3,74 & 0,54 & 14,41 \\
\hline
\end{tabular}


2.16 El desarrollo de la Beca como experiencia de aprendizaje me ha $\quad 88 \quad \mathbf{3 , 7 4} \quad 0,58 \quad 15,44$ aportado elementos que claramente pueden mejorar mi desarrollo como docente de EBA.

\begin{tabular}{|c|c|c|c|c|}
\hline $\begin{array}{l}2.17 \text { Los elementos aportados desde el Seminario pueden ser } \\
\text { transferidos al contexto de mi CEBA. }\end{array}$ & 88 & 3,60 & 0,60 & 5,59 \\
\hline $\begin{array}{l}\text { 2.18 Las redes de trabajo creadas en este viaje de aprendizaje han } \\
\text { potenciado los aprendizajes desarrollados durante el Seminario. }\end{array}$ & 88 & 3,52 & 0,68 & 19,24 \\
\hline $\begin{array}{l}\text { 2.19 Las redes de trabajo generadas en este viaje de aprendizaje podrán } \\
\text { potenciar el desarrollo de los CEBAs en el Perú. }\end{array}$ & 88 & 3,57 & 0,66 & 18,42 \\
\hline & 88 & 3,62 & 0,46 & 13,77 \\
\hline
\end{tabular}

En el caso de la satisfacción general sobre elementos contextuales y metodología todas las valoraciones han superado los 3 puntos, lo cual se considera como muy adecuado. Sin embargo, los ítems que obtienen mayor puntuación son el 2.15 (La defensa del proyecto como elemento de socialización) y el 2.16 (El desarrollo de la Beca como experiencia de aprendizaje me ha aportado elementos que claramente pueden mejorar mi desarrollo como docente de EBA).

\subsection{Resultados análisis estadísticos. Valoraciones cuantitativas (2)}

Para poder analizar las diferencias estadísticamente significativas a partir de los resultados descriptivos para cada bloque, presentamos a continuación los resultados sobre los distintos análisis de datos realizados.

Por un lado, se haya la prueba W de Kendall para observar presencia o ausencia de diferencias significativas entre las variables de la dimensión 1 del cuestionario, así como entre sus totales; todo ello realizado por parejas de ítems.

Tabla 5. Diferencias bloque 1. Actividades conferencias y experiencias

\begin{tabular}{|lll|}
\hline Ítems & W Kendall & sig.asintót. \\
\hline $\mathbf{1 . 1 - 1 . 4}$ & 0,051 & $\mathbf{0 , 0 3 4}$ \\
$\mathbf{1 . 2 - 1 . 5}$ & 0,003 & 0,622 \\
$\mathbf{1 . 3 - 1 . 6}$ & 0,034 & 0,083 \\
$\mathbf{t 1 - t 2}$ & 0,022 & 0,166
\end{tabular}

Como podemos observar en la tabla 5, únicamente encontramos diferencias estadísticamente significativas entre la primera pareja de ítems, que se refieren "la relevancia respecto a mi desarrollo como docente EBA" respecto a las conferencias (ítems 1.1) y en cuanto a las experiencias educativas sobre las visitas (ítem 1.4). 
Por otro lado, analizamos las diferencias para cada ítem de la dimensión 1 sobre los cursos. Es decir, para cada curso se busca la ausencia o presencia de significaciones entre ambos criterios analizados en cada caso -de relevancia y transferibilidad-.

Tabla 6. Diferencias bloque 1. Cursos

\begin{tabular}{lll} 
Ítems & W Kendall & sig.asintót. \\
\hline $\mathbf{1 . 7}$ & 0,189 & $\mathbf{0 , 0 0 0}$ \\
$\mathbf{1 . 8}$ & 0,166 & $\mathbf{0 , 0 0 0}$ \\
$\mathbf{1 . 9}$ & 0,191 & $\mathbf{0 , 0 0 0}$ \\
$\mathbf{1 . 1 0}$ & 0,100 & 0,366 \\
$\mathbf{1 . 1 1}$ & 0,063 & $\mathbf{0 , 0 2 1}$ \\
$\mathbf{1 . 1 2}$ & 0,094 & $\mathbf{0 , 0 0 5}$ \\
$\mathbf{1 . 1 3}$ & 0,053 & $\mathbf{0 , 0 3 3}$ \\
$\mathbf{1 . 1 4}$ & 0,042 & 0,058 \\
$\mathbf{1 . 1 5}$ & 5,333 & $\mathbf{0 , 0 2 1}$ \\
$\mathbf{1 . 1 6}$ & 0,019 & 0,206 \\
$\mathbf{1 . 1 7}$ & 0,016 & 0,248 \\
\hline $\mathbf{1 . 1 8}$ & 0,053 & 0,034 \\
\hline
\end{tabular}

En cuanto al grupo de ítems anteriormente analizados, podemos observar en la tabla 6 que existen diferencias estadísticamente significativas en la mitad de los cursos. Por lo tanto, podemos afirmar que la relevancia puntuada sobre un curso no se relaciona con una misma transferibilidad, y viceversa. Si no que, existen diferencias estadísticamente significativas entre ambos criterios para el ítem 1.7 (Bases para la educación de personas jóvenes y adultas), 1.8 (Políticas educativas y documentos internacionales), 1.9 (Evaluación en educación personas jóvenes y adultas), 1.11 (Teorías de aprendizaje), 1.12 (Alfabetización), 1.13 (Enfoque de competencias y currículum), 1.15 (Materiales didácticos. Creación y análisis) y 1.18 (Diseño de proyecto. Taller de proyecto). Sin embargo, no existen diferencias significativas entre la relevancia de algunos cursos y su transferibilidad, como es el caso del 1.10 (Diagnóstico y evaluación de proyectos), 1.14 (Estrategias e-a. APB / Expositivo / Cooperativo), 1.16 (Estilos de aprendizaje. Inteligencia emocional) y 1.17 (Estilos de aprendizaje. Inteligencias múltiples).

\subsection{Resultados cualitativos}

La valoración se ha realizado de acuerdo a dos preguntas abiertas sobre el cuestionario de satisfacción implementado para poder valorar de manera más profunda la opinión.

- ¿Qué elementos destacarías como muy positivos?, ¿qué aprendizajes más destacados? 
- ¿Qué sugerencias de mejora podrías aportar?

Todos los resultados se presentan en relación a un análisis de contenido sobre los aspectos más destacados y los aspectos a mejorar, de acuerdo a su frecuencia de aparición ordenados de mayor a menor:

Tabla 7. Resultados sobre las valoraciones emitidas

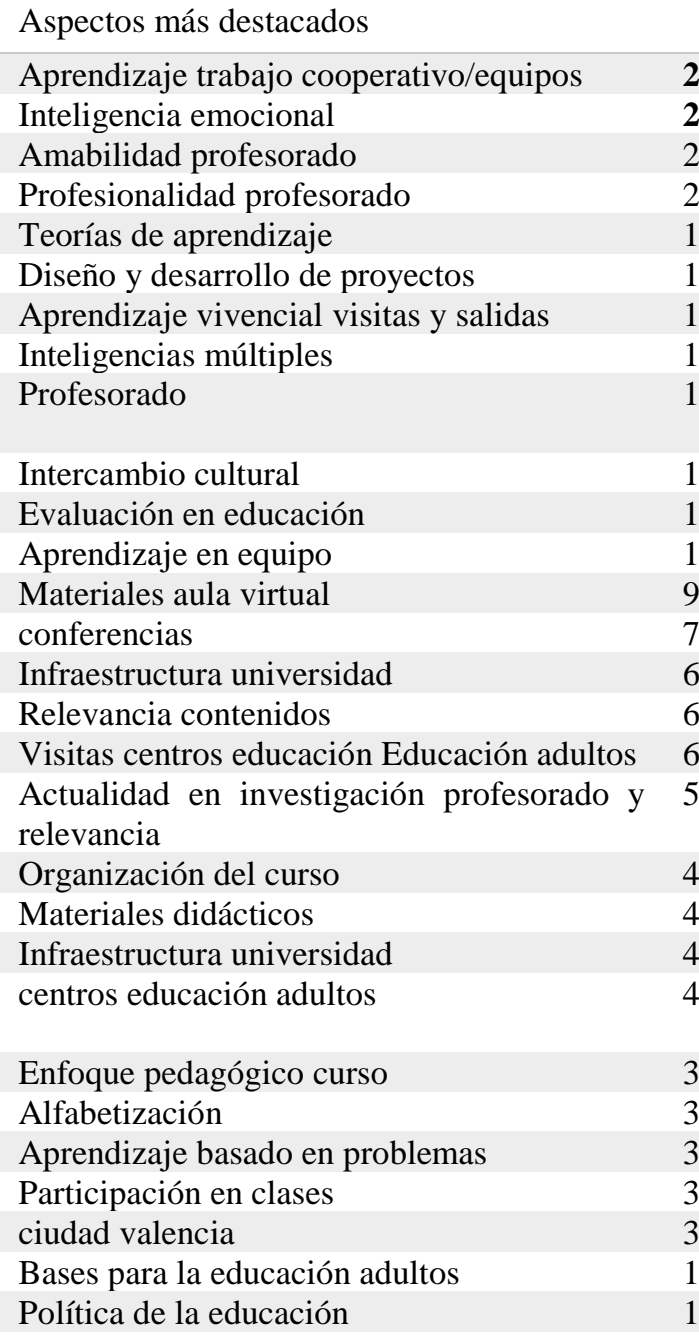

Aspectos a mejorar

27 Reducir horario a lunes y viernes $\quad \mathbf{2 2}$

22 Reducir horario en general 17

21 Ampliar horario en el tiempo 15

20 Más actividades prácticas en el aula 13

19 Contextualizar contenidos 10

17 Ampliar visitas y salidas 10

14 Clases más participativas 7

13 Mejorar acceso internet y computadoras 7

13 Ampliar contenidos sobre educación 4 adultos

10 Facilitar información previamente al curso 4

10 Intercambio de experiencias 4

10 Facilitar dossier de material 3

Mejorar trato trabajadores residencia 3

Reducir distancia entre aulas 3

Evaluar a los y las participantes 3

Respetar cambio horario 2

Mejorar trato personal residencia 2

Más actividades a distancia 1

Mejorar acompañamiento proyectos $\quad 1$

Reducir ratio 1

Mejorar menú residencia $\quad 1$

Distribuir equipos de trabajo de manera 1 heterogénea

En cuanto a los resultados del análisis de contenido realizado sobre los elementos que destacarían como mejor valorados aparece el "Aprendizaje trabajo cooperativo/equipos" (referido a metodología) y la "Inteligencia emocional" (referido a cursos) como lo más adecuado. En el caso de los aspectos a mejorar, aparece "Reducir horario a lunes y viernes" y "Reducir horario en general" como algo a destacar.

\subsection{Resultados valoraciones creativas personales (murales y fotografías)}


Asimismo, se realizaron dos dinámicas grupales de evaluación de la satisfacción mediante murales (mural 1 conferencias-visitas-salidas; mural 2 cursos; mural 3 grupos; y mural 4 otros) y fotos (más representativas del curso).

Todos los resultados se presentan en relación a un análisis de contenido y categorización de los comentarios sobre los aspectos más destacados y los aspectos a mejorar (en el caso de los murales), y sobre los aspectos más relevantes (en el caso de las fotografías), de acuerdo a su frecuencia de aparición ordenada de mayor a menor:

Tabla 8. Resultados sobre los murales creados

MURAL 1: Conferencias-visitas-salidas

\begin{tabular}{llll}
\hline Aspectos más destacados & & Aspectos a mejorar \\
Aprendizaje vivencial en visitas & $\mathbf{6}$ & Mejor participación en conferencias & $\mathbf{2}$ \\
Relevancia visitas & $\mathbf{6}$ & Más actividades vivenciales en el aula & $\mathbf{2}$ \\
Intercambio cultural & 2 & Ampliar tiempo de visitas y salidas & $\mathbf{2}$ \\
Calidad conferencias & 2 & ampliar tiempo de conferencias & 1 \\
Conferencias motivadoras & 1 & más visitas centros formación & 1 \\
Intercambio de experiencias & 1 & & \\
Conocer centros formación adultos & 1 & &
\end{tabular}

MURAL 2: Cursos

\begin{tabular}{llll}
\hline Aspectos más destacados & & Aspectos a mejorar \\
& & & 5 \\
Profesionalidad profesorado & $\mathbf{8}$ & Ampliar horario en el tiempo & 3 \\
Relevancia de contenidos & 5 & Mejorar horario & 3 \\
Trabajo cooperativo & 2 & Más aprendizaje en centros & 3 \\
Metodología participativa & 2 & Más estrategias y técnicas formación adultos & 2 \\
Enriquecimiento de contenido & 2 & Mejorar participación en cursos & 1 \\
Actitud docente & 2 & Evaluar a los y las participantes & 1 \\
Enriquecimiento cultural & 1 & Reducir ratio & \\
Relevancia profesorado & 1 & & \\
Aprendizaje práctico & 1 & &
\end{tabular}

MURAL 3: Grupos

\begin{tabular}{llll}
\hline Aspectos más destacados & & Aspectos a mejorar & \\
Trabajo en equipo & $\mathbf{6}$ & Ampliar horario en el tiempo & $\mathbf{4}$ \\
\hline Metodologías en investigación & 4 & Mejorar ritmo de diferentes grupo & $\mathbf{4}$ \\
Utilidad proyecto & 3 & Contextualizar contenidos & 2 \\
\hline Aprendizaje práctico & 2 & & \\
Intercambio de experiencias & 2 & & \\
Acompañamiento permanente & 2 &
\end{tabular}

MURAL 4: Otros

$\begin{array}{llll}\text { Aspectos más destacados } & & \text { Aspectos a mejorar } & \\ \text { Intercambio cultural } & \mathbf{4} & \text { Mejorar acceso biblioteca } & \mathbf{4} \\ \text { Adecuado ambiente trabajo } & 1 & \text { Reducir horario a lunes y viernes } & \mathbf{4} \\ \text { Amabilidad profesorado } & 1 & \text { Mejorar intercambio experiencias } & 1 \\ \text { Excelente organización } & 1 & \text { Mejorar horario } & 1\end{array}$


En cuanto a los resultados del análisis de contenido realizado sobre los elementos que destacarían como mejor valorados, en el caso de los murales desarrollados, aparece en el mural 1 el "Aprendizaje vivencial en visitas" y la "Relevancia visitas" como lo más destacado. Sin embargo, en aspectos a mejorar aparecen los más destacados como "Mejor participación en conferencias", "Más actividades vivenciales en el aula" y "Ampliar tiempo de visitas y salidas". Sobre los resultados del mural 2, aparece la "Profesionalidad profesorado" como lo más destacado y "Ampliar horario en el tiempo" en propuestas de mejora. En relación al mural 3 se destacan mayormente el "Trabajo en equipo" y se deben mejorar de manera mayor "Ampliar horario en el tiempo" y "Mejorar ritmo de diferentes grupo". Si nos fijamos en los resultados del mural 4, el "Intercambio cultural" es lo más destacado y como aspectos mayormente a mejorar aparecen "Mejorar acceso biblioteca" y "Reducir horario a lunes y viernes".

Tabla 9. Resultados sobre las fotos más relevantes

\section{FOTOS}

\begin{tabular}{ll}
\hline Aspectos más relevantes & 13 \\
\hline Profesorado & 11 \\
\hline Trabajo en equipo & 8 \\
\hline Salidas y visitas & 7 \\
\hline Diseño y desarrollo de proyectos & 6 \\
\hline Conferencias & 5 \\
\hline Exposición de proyectos & 5 \\
Participación en clase & 4 \\
Ciudad de Valencia & 4 \\
Centros formación adultos & 4 \\
\hline Infraestructura universidad &
\end{tabular}

A partir de los resultados del análisis sobre las fotografías seleccionadas por los y las participantes como las más relevantes, podemos deducir que se relacionad de manera más destacada con el "Profesorado" y el "Trabajo en equipo".

\section{Conclusiones}

Los resultados, en general, son adecuados en ambos sentidos en todos los casos estudiados. La evaluación de producto realizada ha sido muy enriquecedora para poder tomar decisiones posteriores a su realización y seguir avanzando en la mejora de este tipo de programas de formación de profesorado. 
Por ello, al finalizar el programa y haber desarrollado la evaluación de producto, a la vista de los resultados obtenidos es el momento adecuado para poder tomar decisiones que nos permitan, tanto presentar datos analizados en cuanto a elementos positivos y negativos, así como continuar con algunos aspectos deseados o modificar o readaptar otros que no han sido adecuados (Jiménez, 1999).

También, la evaluación realizada, nos ha permitido conocer la satisfacción del profesorado participante, observando en general, resultados adecuados.

Por lo tanto, en términos evaluativos sobre el programa, desde el análisis de criterios en relación a la eficacia, eficiencia, satisfacción de los implicados, impacto y funcionalidad del programa (Perales, Ortega y Jornet, 2013), podemos encontrar elementos, anteriormente presentados, que nos garantizan su adecuación.

\section{Referencias}

Bericat, E. (1998). La integración de los métodos cuantitativo y cualitativo en la investigación social. Significado y medida. Barcelona: Ariel.

Dray, B. J., Lowenthal, P. R., Miszkiewicz, M. J., Ruiz-Primo, M. A., y Marczynski, K. (2011). Developing an instrument to assess student readiness for online learning: A validation study. Distance Education, 32(1), 29-47.

Jiménez, B. (1999). Evaluación de Programas, Centros y Profesores. Madrid: Síntesis.

Jornet, J. M., González-Such, J., y Perales, Mª J. (2013). Investigación evaluativa: Una perspectiva basada en la complementariedad metodológica (cuantitativacualitativa). Perú: CREA.

Jornet, J.M. (2007). La evaluación de los aprendizajes universitarios. Ponencia invitada en las III Jornadas de intercambio de grupos de formación del profesorado de la Universidad de Cádiz. UCA-Publicaciones: Cádiz.

Jornet, J.M., y González Such, J. (2009). Evaluación criterial: determinación de estándares de interpretación (EE) para pruebas de rendimiento educativo. Estudios sobre Educación, 16, 103-123.

Jornet, J.M., González-Such, J., y Perales, Mª J. (Eds.) (2013). Investigación evaluativa: Una perspectiva basada en la complementariedad metodológica (cuantitativacualitativa). Perú: CREA. 
Paiba, M. (2015). Propuestas de medidas de política para superar los nudos críticos de la Educación Básica Alternativa (EBA). En Venegas, D. Educación de jóvenes y adultos a lo largo de la vida. Lima: EDAPROSPO.

Perales, M. J., Ortega, S. y Jornet, J. M. (2012). La evaluación como condición de calidad en la educación intercultural. En L. Díe (Coord.). Aprendiendo a ser iguales. Manual de Educación Intercultural. Valencia: CEIMIGRA.

Pérez-Juste, R. (2000). La evaluación de programas educativos: conceptos básicos, planteamientos generales y problemática. Revista de Investigación Educativa, 2000, 18(2), 261-287.

Sánchez-Rodríguez, L. (2015). Evaluación creativa. Tesis doctoral. Madrid: Universidad Complutense.

Scriven, M. (1967). The methodology of evaluation. En R. W. Tyler, R. M. Gagné, y M. Scriven (Eds.), Perspectives of curriculum evaluation, 39-83. Chicago: Rand McNally.

Stufflebeam, D.L. y Shinkfiel, A.J. (1987). Evaluación sistemática. Guía teórica y práctica. Barcelona: Paidós-MEC.

Venegas, D. (2015). Educación de jóvenes y adultos a lo largo de la vida. Lima: EDAPROSPO. 